\title{
A Registration Method for Multimodal Medical Images Using Contours Mutual Information
}

\author{
Ying Qian \\ The lab of Graphics and Multimedia \\ Chongqing University of Posts and Telecommunications \\ Chongqing, China
}

\begin{abstract}
In recent years, mutual information has developed as a popular image registration measure especially in multimodality image registration. For different modality medical images, the contour of tissues or organs is similarity. In this paper, an effective new registration method of the multimodal medical images based on the contour mutual information is proposed. Firstly, get the contour through variational level set method. Secondly, within the contour pixels are assigned the same grayscale value, obtain two contour images. Finally, two contour images using mutual information as similarity measure for image registration. The experiment results show that the registration algorithm proposed in this paper can do more effectively and more accurately work than normalized mutual information and gradient mutual information. (Abstract)
\end{abstract}

Keywords - contour mutual information; mutual information; multimodal medical image; image registration; variational level set method

\section{INTRODUCTION}

In actual clinical diagnosis, a single modal of image usually unable to provide enough information to doctors. So we need to have the step of different modal image fusion, thus providing more information to doctor, and then they can understand the comprehensive information from pathological tissue or organs, make better medical diagnosis. Moreover, image registration is a premise key in image fusion, so the multimodal medical image registration becomes a research hotspot in the field of medical image processing. Medical image registration is the ascertaining process of spatial mapping between two images that differs in image acquisition time, image properties, or viewpoint and subsequently producing a result image that is informative. At present, medical image registration methods can be classified as feature-based and intensity-based [1]. In intensity-based registration, mutual information (MI) is one of the intensity based measure, and does not require the definition of landmarks or features such as surfaces [2]. It is an automatic method and it applies to a variety of modal image registration [3].

The multimodal image registration method based on mutual information of image gray intensity, which is no need for us to preprocess. In order to improve the accuracy of the registration, so many scholars made deep researches and improved the registration methods.

\author{
Meng Li, Qingjie Wei, Xuemei Ren \\ The lab of Graphics and Multimedia \\ Chongqing University of Posts and Telecommunications \\ Chongqing, China
}

Studholme [4] proposed a method based on normalized mutual information. But the method ignores the spatial information. Pluim [5] proposed to include spatial information by multiplying MI measure with a gradient term based on the magnitude and the orientation of the gradients. But gradient is sensitive to noise, thus the success rate become lower when images contains noise. Butz and Thiran [6] used mutual information in edge computing. But the edge information is sparse and the mutual information is sensitive to the number of sampling point, thus rendering appear local maximum and result in mismatching. Loeckx [2] proposed a method based on conditional mutual information, spread gray joint histogram from two-dimension to three-dimension. But the method has high computational cost. Ruecker [7] combined the mutual information with image's texture information which be described as gray level co-occurrence matrix. But this method assume that image's texture information have similarity when organ in different modal images.

This paper uses the characteristic that the contours of different modal medical images have similarity, proposed a registration method based on contour mutual information(CMI), extracts contours effectively from images which to be registered, and within the contour pixels are assigned the same gray-scale, That is, contour image. Finally, two contour images using mutual information as similarity measure for image registration. CMI efficiently solves problems of poor robustness and low accuracy in image registration.

\section{MUTUAL INFORMATION}

\section{A. Definition}

MI is selected as the similar measure. MI is an important concept of information theory which is used to represent the statistics correlation of two sets of data which has been widely used in the image registration. Starting from a reference image $X$ and floating image $Y$ with intensity bins $x$ and $y$, the calculation of MI of two images $X$ and $Y$ is as follows:

$$
\begin{gathered}
\mathrm{I}(X, Y)=H(X)+H(Y)-H(X, Y) \\
H(X, Y)=-\sum_{x, y} p(x, y) \log (p(x, y)) \\
H(X)=-\sum_{x} p(x) \log (p(x)) \text { the joint and marginal entropy of }
\end{gathered}
$$$$
\text { random variables } X \text { and } Y \text {. }
$$ 


\section{B. Problems}

Mutual information registration method based on gray value is a statistical method for registration, has nothing to do with the pixel location. Constructs a size for the test image A $(256 * 256)$, pixels of each column from top to bottom, with gray value from 0 to 255 , as shown in Fig.1(a), A rotate $180^{\circ}$, obtain test image B, as shown in Fig.1(b). A is the reference image, $\mathrm{B}$ is the floating image. Floating image $360^{\circ}$ rotation around itself(from $-180^{\circ}$ to $180^{\circ}$ ), Observe the reference image and the floating image of the mutual information curve, Respectively, at $0^{\circ}$ and $180^{\circ}$, the maximum mutual information appears, as shown in Fig.1(c).

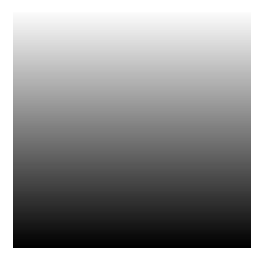

(a)reference A

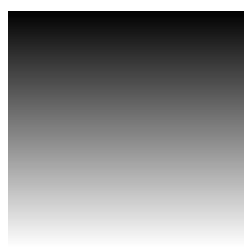

(b)floating image B

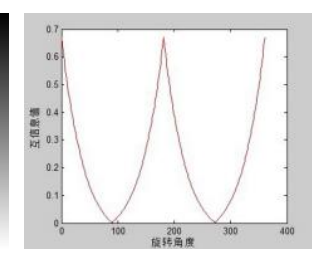

(c)mutual information curve
Fig. 1. Test images and mutual information curve.

The research direction of many researchers is how the spatial information of image is introduced to the mutual information to produce a new similarity measure. But the robust is not very good; the main reasons are the following:

- As shown in Fig.2(a), image with noise, weaken the correlation between images, easy to fall into local extreme value leads to wrong registration;

- As shown in Fig.2(b), if the image appears to be missing which make registration less effective, the registration effect will be worse;

- As shown in Fig.2(c), due to less spatial resolution and image information content, the image of low resolution images can lead to the problem of robust ;

- Images to be registered have less effective information, such as the top of the skull, as shown in Fig.2(d).

In this paper, analyzes the robustness of registration according to mutual information measure curve. In Fig.1, the abscissa of mutual information is the rotation angle $\left(-25^{\circ}\right.$ to $25^{\circ}$ ), the vertical axis is the amount of the mutual information. As can be seen from the figure, there are have different degrees of local maxima in the four cases mentioned above.
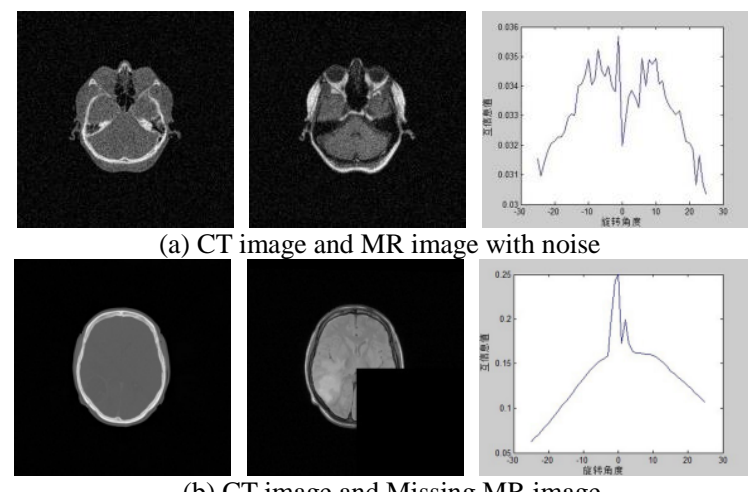

with noise

(b) CT image and Missing MR image
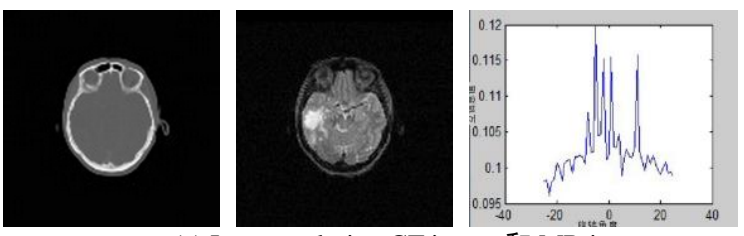

(c) Low-resolution CT image 和 MR image
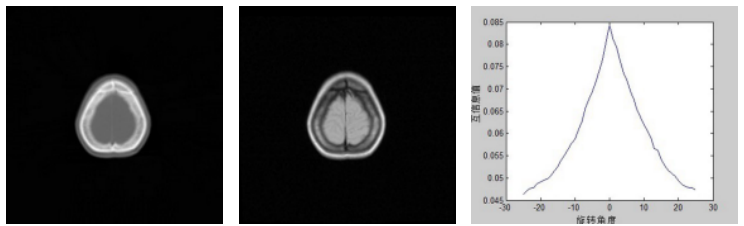

(d) CT skull image 和 MR skull image

Fig. 2. Mutual information curves under different circumstances.

Although some of the improved algorithm considers the spatial information of the image, but have a major impact on the registration is still gray values, its spatial information is essentially the use of the gray information, because of the limitations are not widely used.

\section{METHOD}

Contour as a stable characteristic of image, when the image translating or rotating, which can keep better corresponding relation. So before get contour image, we should extract contours of image.

The contour extraction method will choose an operator to detect the image edge which based on the image edge features. Then we will connect the edge point into a closed curve to obtain the target contour.

Commonly used methods of edge detection include: Roberts operator, Sobel operator, Prewitt operator, etc. But these edge detection methods are sensitive to noise, and will enhance the noise in the edge detection. This paper mainly use feature of contour, so effectively getting contour of image is the key of CMI.

In this paper, we adopt a variational level set method proposed by Li [8], which have no need to reinitialize. The method bring penalty term in energy function, thus ensuring level set function keep become sign function in evolutionary process , and it avoids reinitialize in the process. After get contours of two images, filling contour unified gray value and then get contour image.

CMI has two advantages, firstly, ensures the strict corresponding relations exist between two contours images, add gray-scale correlation between images. Secondly, it used spatial information that contours in different modal images have similarity.

The following describes specific steps which about a way of image registration based on contour mutual information:

1) Two images are given to be registered, and they are reference image $R$ and floating image $F$.

2) Get contours of two images by variational level set method, and then fill unified gray value to get contour image $\left(R^{\prime}\right.$. $\left.F^{\prime}\right)$. 
3) Mutual information be used as measure function, and Powell optimization algorithm execute optimization methodology of parameters for image $R$ ' and $F$ '. The process of optimization uses the nearest neighbor interpolation algorithm.

4) Optimal spatial transform parameters reform the floating image $F$ according to the Powell algorithm, obtain registration results.

\section{EXPERIMENT}

In this paper, the experiment data are come from Retrospective Registration Evaluation Project of the Vanderbilt University, and the images resolution is $256 \times 256$. The experiment 1 validates the robustness of CMI, the rest experiments were compared with Gradient Mutual Information (GMI, from reference [3]) and Normalized Mutual Information (NMI, from reference [4]).

\section{A. Experiment 1 Validate the Robustness}

According to mutual information curve from Fig.3, using image mutual information method can lead peak value become not so sharp. However, the method proposed in this paper has a sharp peak, and it is easy to detect the best position of registration. Meanwhile, it is not easy to appear the phenomenon of local maximum because of the smooth curve.

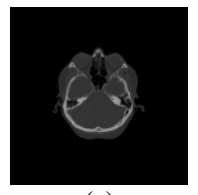

(a)

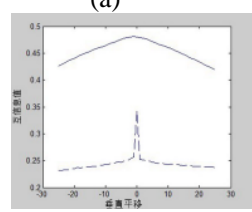

(e)

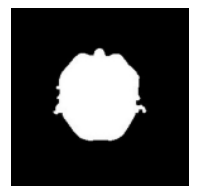

(b)

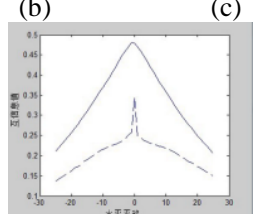

(f)

(c)
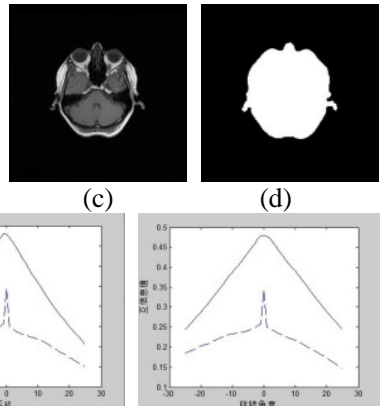

(g)
Fig. 3. The results of robustness of experiments.

(a) is CT original registration image and (b) is MR original registration image, (c)is CT contour image and (d) is MR contour image. (e)is mutual information curve based on image gray value and the method of paper to rotate the images (from $-25^{\circ}$ to $25^{\circ}$ ). (f) is mutual information curve based on image gray value and the method of paper for vertical translation (from -25 To 25 pixels). (g) is mutual information curve based on image gray value and the method of paper for horizontal translation. The solid line is the measure of the curve based on image gray value and the dashed line is the measure of the curve based on the contour mutual information.

In order to validate the robustness of the registration algorithm based on contour mutual information, using the method of this thesis to create mutual information curve, as Fig.4. Compared the curve of Fig.2, the mutual information curve created by CMI has good robustness.

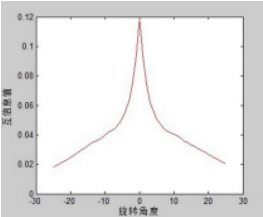

(a)

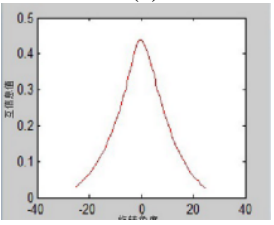

(c)

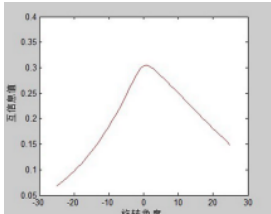

(b)

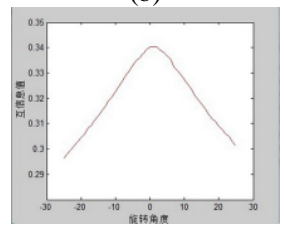

(d)
Fig. 4. Measure curve based on contour mutual information.

(a)(b)(c)(d)Corresponding to CMI of test image in Fig.1 mutual information measure curve.

\section{B. Experiment 2 Validate the Accuracy}

As shown in Fig.5, the edge images of CT are applied to the MR images, by this to detect the effect of registration. The bone tissue in MR images are close to the background grayscale value, However, it have large different with the background gray-scale value of CT image, thus leading to registration errors which use the method of mutual information. As shown in Fig.5 (d); The gradient mutual information, due to considerate the information of space and orientation, the registration results have more advantages compare to the method which based on normalized mutual information; From the Fig.5 (e), there are many errors at edges, but effects obtained by CMI show that the image has been substantially aligned at edges after registration.

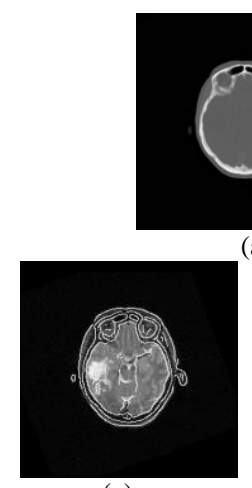

(c)
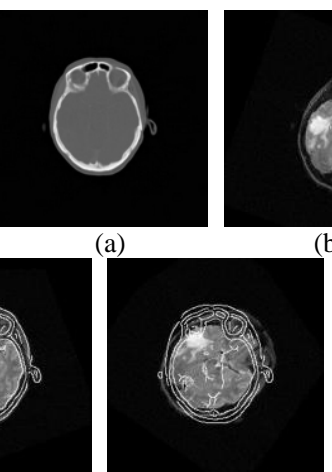

(d)
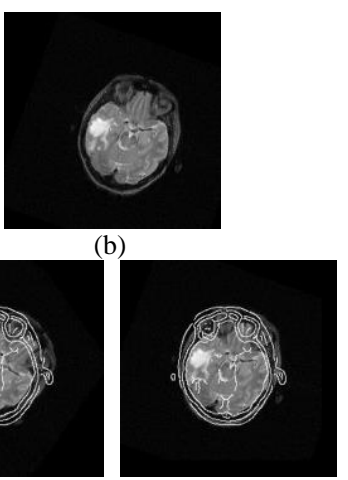

(e)
Fig. 5. Experimental results verify the accuracy of CMI.

\section{Experiment 3 Validate the robustness when Image have Missing information}

From Fig.6 (a), CT image are mainly reflects the skeletal structure, its gray-level changes small. 
We mainly use edges information to register with MR image. When Image has large missing information, it will mismatch easier. From experimental results, CMI has obvious advantages compared with other two methods in gray-scale difference.
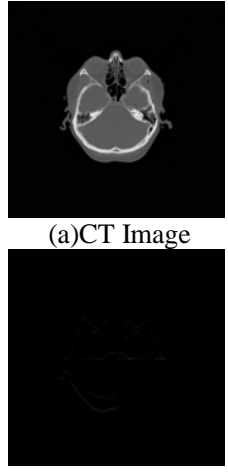

(d)CMI
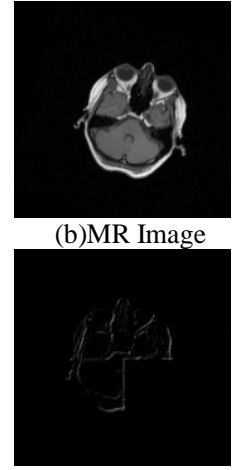

(e)NMI
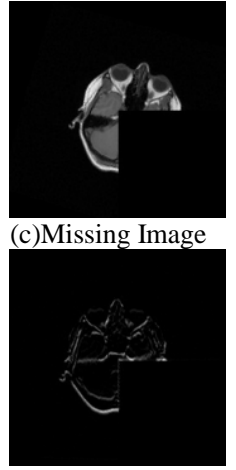

(f)GMI
Fig. 6. The results on the robustness of image deletion.

(a)(b)(c)are CT images and MR image in the same layer from the head of a patient. (a) is original CT image (b) is original MR (T2-weighted) image, (c) have missing (Setting the region of the image's gray value to 0 to simulate the effect of information lacking). (d)(e)(f)corresponding to the result of the registration image obtained by CMI, NMI and GMI which subtract registration images.

\section{Experiment 4 validate the robustness when image has noise}

The gradient is sensitive to noise, when image have a little of noise, the registration method which based on gradient mutual information will easily appear local maximum, it will results in mismatching. As shown in Fig.7 (a), this paper choose three groups of head images in CT modal and MR modal (T1 weighted, T2 weighted, PD weighted), which have been registered and in the corresponding layer. As shown in Fig.7 (a), add noise in CT and MR images. Noised image of CT as a reference image, let the MR images horizontal, vertical position and angle transformation from $\left(-25,-25,-25^{\circ}\right)$ to $(25$, $\left.25,25^{\circ}\right)$ get floating images, to get $50 \times 3$ groups of experimental subjects. Three parameters among them are the horizontal of translation (pixel), vertical translation (pixel), rotation angle. Results from three methods are shown in Table1. From data in the Table1we can see the registration of the CMI is more accurate than other two methods. In the CT image and PD image registration, the noise will make impact on gradient mutual information, thus making the effect of registration unsatisfactory.

TABLE I. THE EXPERIMENTAL ReSUlts

\begin{tabular}{|c|c|c|c|}
\hline \multirow{2}{*}{ Experimental object } & \multicolumn{3}{|c|}{ Robustness verification method for noisy image } \\
\cline { 2 - 4 } & CMI & NMI & GMI \\
\hline CT-T1 & $86 \%$ & $80 \%$ & $80 \%$ \\
\hline CT-T2 & $86 \%$ & $82 \%$ & $78 \%$ \\
\hline CT-PD & $90 \%$ & $84 \%$ & $82 \%$ \\
\hline
\end{tabular}
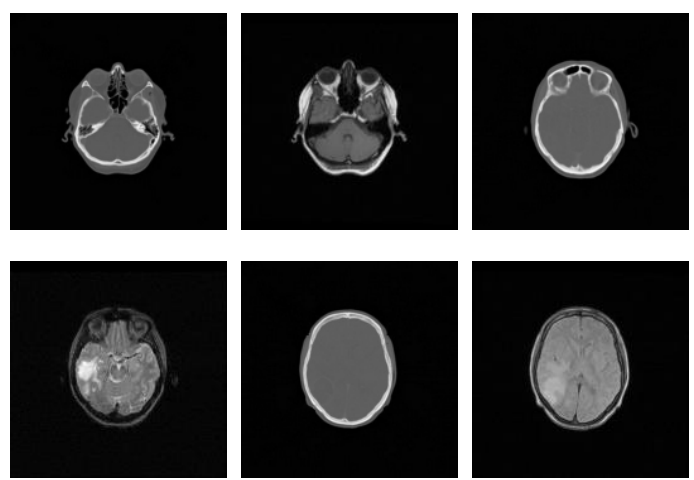

(a) CT and MR

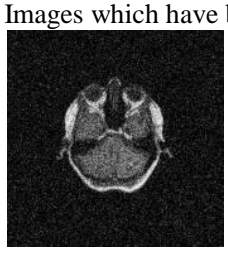

been registrated
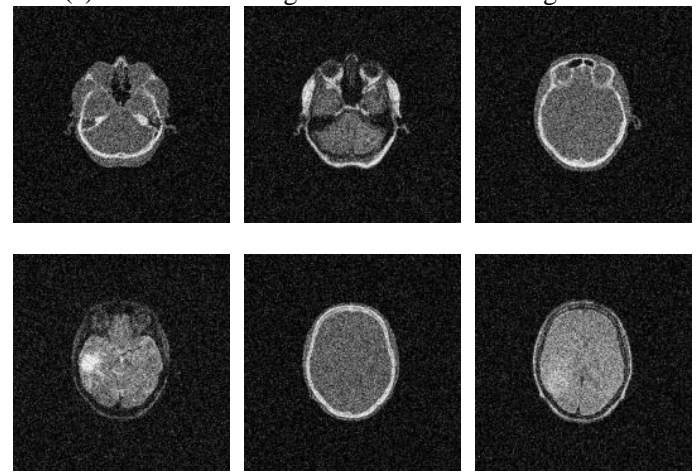

(b) Images are added $40 \%$ random noise which comes from (a)

Fig. 7. Robustness verification method for noisy image.

\section{CONCLUSIONS}

Recently, the algorithm in multimodality medical image registration has poor robust and accuracy. This paper proposed a registration method based on contour mutual information. On the one hand, it uses a spatial characteristic, that the contours of tissue and organs in different modal have similarity. On the other hand, the method that fill the same gray values to the contour, which can add the correlation information among contour image and reduce the interference in image registration when image have missing information, thus improving precision of registration. The experiments show that the method of this paper can achieve more accurate registration results. But this paper has not considered nonlinear registration in multimodality medical image. We will make more research in further work.

\section{REFERENCES}

[1] Zitova B, Flusser J, Image registration methods a survey, Image and vision computing, pp: 977-1000, 2003.

[2] Loeckx D, Slagmolen P, Maes F, Vandermeulen D, Suetens P, Non-rigid image registration using conditional mutual information, IEEE transactions on medical imaging, pp:19-29, 2010.

[3] Nigris D, Collins D, Arbel T, Multi-modal image registration based on gradient orientations of minimal uncertainty, IEEE transactions on medical imaging, pp:2343-2354, 2012.

[4] Studholme C, Hill D L G, Hawkes D J, An overlap invariant entropy measure of 3D medical image alignment, Pattern recognition, pp: $71-86$, 1999.

[5] Pluim J P, Maintz J B, Viergever M A, Image registration by maximization of combined mutual information and gradient information, IEEE transactions on medical imaging, pp: 809-814,2000 
[6] Butz T, Thiran J P, Affine registration with feature space mutual information, MICCAI 2001, LNCS 2208. 549-556.

[7] Rueckert D, Clarkson M J,Hill D L G, Hawkes D J, Non-grid registration using higher order mutual information, Proceeding of SPIE Medical Imaging 2000 : Image Processing, SanDiego, pp:438-447, 2000.
[8] Li CM, Xu CY, Gui CF, et,al. Level set evolution without reinitialization: A new variational formulation. In: Schmid C, Soatto S, Tomasi C, eds. Proc. of the 2005 IEEE Computer Society Conference. on Computer Vision and Pattern Recognition. Washington: IEEE Computer Society Press, pp: $430-436,2005$. 